\title{
A one-sided theorem for the product of Abel and Cesáro summability methods
}

\author{
Yllmaz Erdem and İbrahim Çanak
}




\title{
A ONE-SIDED THEOREM FOR THE PRODUCT OF ABEL AND CESÀRO SUMMABILITY METHODS
}

\author{
YILMAZ ERDEM AND İBRAHIM ÇANAK
}

Received 09 May, 2014

\begin{abstract}
In this paper, a one-sided condition is given to recover $(C, \alpha)$ summability of a sequence from its $(A)(C, \alpha+1)$ summability. Our result extends and generalizes the well known classical Tauberian theorems given for Abel and Cesàro summability methods.
\end{abstract}

2010 Mathematics Subject Classification: 40E05; 40G05; 40G10

Keywords: Abel summability, Cesàro summability, $(A)(C, \alpha)$ summability, $(C, \alpha)$ summability, Tauberian conditions and theorems, product methods

\section{INTRODUCTION}

Let $\sum_{n=0}^{\infty} a_{n}$ be an infinite series of real numbers with partial sums $s_{n}=\sum_{k=0}^{n} a_{k}$. For all nonnegative integers $m$, we define

$$
(n \Delta)_{m} s_{n}=n \Delta\left((n \Delta)_{m-1} s_{n}\right),
$$

where $(n \Delta)_{0} s_{n}=s_{n}$ and $(n \Delta)_{1} s_{n}=n \Delta s_{n}$.

The backward difference $\Delta s_{n}$ of $s_{n}$ is defined to be $\Delta s_{n}=s_{n}-s_{n-1}, n \geq 1$, with $\Delta s_{0}=s_{0}$.

Let $A_{n}^{\alpha}$ be defined by generating function $(1-x)^{-\alpha-1}=\sum_{n=0}^{\infty} A_{n}^{\alpha} x^{n}(|x|<1)$, where

$$
A_{0}^{\alpha}=0, \quad A_{n}^{\alpha}=\frac{\alpha(\alpha+1) \cdots(\alpha+n)}{n !}=\frac{\Gamma(n+\alpha+1)}{\Gamma(n+1) \Gamma(\alpha+1)}
$$

for $\alpha>-1$.

A sequence $\left(s_{n}\right)$ is said to be summable by the Cesàro mean of order $\alpha$, or $(C, \alpha)$ summable to $s$, where $\alpha>-1$, and we write $s_{n} \rightarrow s(C, \alpha)$ if

$$
s_{n}^{\alpha}=\frac{S_{n}^{\alpha}}{A_{n}^{\alpha}}=\frac{1}{A_{n}^{\alpha}} \sum_{k=0}^{n} A_{n-k}^{\alpha-1} s_{k} \rightarrow s
$$

as $n \rightarrow \infty$.

We write $\tau_{n}=n a_{n}$ and denote the $(C, \alpha)$ mean of $\left(\tau_{n}\right)$ by $\tau_{n}^{\alpha}$. Borwein [4] showed that if a sequence is $(C, \alpha)$ summable to $s$ for any $\alpha>-1$, it is $(C, \beta)$ summable to 
$s$ for any $\beta>\alpha$. It is also well known that the $(C, \alpha)$ summability method is regular (see [3]). Note that $(C, 0)$ summability reduces to the ordinary convergence.

A sequence $\left(s_{n}\right)$ is said to be Abel summable to $s$, and we write $s_{n} \rightarrow s(A)$ if the series $\sum_{n=0}^{\infty} a_{n} x^{n}$ is convergent for $0 \leq x<1$ and tends to $s$ as $x \rightarrow 1^{-}$. It is well known that if a sequence is $(C, \alpha)$ summable to $s$ for any $\alpha>-1$, then it is Abel summable to $s$ (see [2]).

A sequence $\left(s_{n}\right)$ is said to be $(A)(C, \alpha)$ summable to $s$, and we write $s_{n} \rightarrow$ $s(A)(C, \alpha)$ if the series $\sum_{n=0}^{\infty}\left(s_{n}^{\alpha}-s_{n-1}^{\alpha}\right) x^{n}$, with $s_{-1}^{\alpha}=0$, is convergent for $0 \leq$ $x<1$ and tends to $s$ as $x \rightarrow 1^{-}$. Note that $(A)(C, \alpha)$ summability reduces to the Abel summability when $\alpha=0$.

The identity $s_{n}-s_{n}^{1}=\tau_{n}^{1}$ is known as the Kronecker identity and it will be used in the proof of the main result. Throughout this paper we use the symbols $s_{n}=o(1)$ and $s_{n}=O(1)$ to mean that $s_{n} \rightarrow 0$ as $n \rightarrow \infty$ and $\left(s_{n}\right)$ is bounded for large enough $n$.

\section{Preliminary RESUlts}

A theorem due to Abel [1] states that if $\left(s_{n}\right)$ converges to $s$, then it is Abel summable to $s$. The converse Abel's theorem is not necessarily true. For example the series $\sum_{n=0}^{\infty}(-1)^{n}$ is not convergent, but it is Abel summable to $1 / 2$. However, the converse of Abel's theorem may be valid under some condition which we call Tauberian condition. Any theorem stating that convergence follows from a summability method and a Tauberian condition is called a Tauberian theorem.

By imposing some restriction on $a_{n}$, Tauber [17] obtained the first partial converses of Abel's theorem.

Theorem 1. If $\left(s_{n}\right)$ is Abel summable to $s$ and $\tau_{n}=o(1)$, then $\left(s_{n}\right)$ converges to $s$.

Theorem 2. If $\left(s_{n}\right)$ is Abel summable to $s$ and $\tau_{n}^{1}=o(1)$, then $\left(s_{n}\right)$ converges to $s$.

Littlewood [12] replaced the condition $\tau_{n}=o(1)$ by $\tau_{n}=O(1)$ and later Hardy and Littlewood [9] obtained the following one-sided Tauberian theorem.

Theorem 3. If $\left(s_{n}\right)$ is Abel summable to $s$ and $\tau_{n} \geq-H$ for some nonnegative constant $H$, then $\left(s_{n}\right)$ converges to $s$.

A generalization of Theorem 3 was given by Szász [16].

Theorem 4. If $\left(s_{n}\right)$ is Abel summable to $s$ and $\tau_{n}^{1} \geq-H$ for some nonnegative constant $H$, then $\left(s_{n}\right)$ is $(C, 1)$ summable to $s$.

Pati [14] have recently obtained more general Tauberian theorems generalizing the classical results for the product of the Abel and $(C, \alpha)$ summability methods. 
Theorem 5. If $\left(s_{n}\right)$ is $(A)(C, \alpha)$ summable to $s$, where $\alpha>0$, and $\tau_{n}^{\alpha} \geq-H$ for some nonnegative constant $H$, then $\left(s_{n}\right)$ is $(C, \alpha)$ summable to $s$.

Theorem 6. The necessary and sufficient condition that the $(A)(C, \alpha+1)$ summability of $\left(s_{n}\right)$ to $s$, where $\alpha>-1$, implies the $(C, \alpha)$ summability of $\left(s_{n}\right)$ to $s$, is that $\tau_{n}^{\alpha+1}=o(1)$.

Tauberian theorems in the sense of Pati were generalized by Çanak et al. [6], Çanak and Erdem [5] and Erdem and Çanak [7]. Çanak et al. [6] proved that if $\left(s_{n}\right)$ is $(A)(C, \alpha)$ summable to $s$ and $(n \Delta)_{m} \tau_{n}^{\alpha+m}=o(1)$ for $m=1,2$, then $\left(s_{n}\right)$ is convergent to $s$. Later, Erdem and Çanak [7] proved the main result in Çanak et al. [6] for all integers $m \geq 1$. Recently, Çanak and Erdem [5] have recovered convergence, $(C, \alpha)$ convergence, and $(C, \alpha)$ slow oscillation of $\left(s_{n}\right)$ depending on the conditions given in terms of $(n \Delta)_{m} \tau_{n}^{\alpha+m}$ for some special cases of $m$.

In this paper, we recover $(C, \alpha)$ convergence of $\left(s_{n}\right)$ from its $(A)(C, \alpha+1)$ summability under the one-sided boundedness of $\left((n \Delta)_{m} \tau_{n}^{\alpha+m}\right)$, where $m \geq 1$ and $\alpha>-1$.

\section{MAin RESUlt}

Our result is based on Theorem 1 and Theorem 3 .

Theorem 7. If $\left(s_{n}\right)$ is $(A)(C, \alpha+1)$, where $\alpha>-1$, summable to $s$, and for some integer $m \geq 0$,

$$
(n \Delta)_{m} \tau_{n}^{\alpha+m} \geq-H
$$

then $\left(s_{n}\right)$ is $(C, \alpha)$ summable to $s$.

From Theorem 7, we deduce the following corollary:

Corollary 1. If $\left(s_{n}\right)$ is $(A)(C, \alpha+1)$, where $\alpha>-1$, summable to $s$, and for some integer $m \geq 0$,

$$
(n \Delta)_{m} \tau_{n}^{m} \geq-H
$$

then $\left(s_{n}\right)$ converges to $s$.

\section{Auxiliary Results}

We need the following lemmas for the proof of Theorem 7.

$\operatorname{Lemma} 1([10,11])$. For $\alpha>-1, \tau_{n}^{\alpha}=n \Delta s_{n}^{\alpha}=n\left(s_{n}^{\alpha}-s_{n-1}^{\alpha}\right)$.

Lemma 2 ([8,11]). For $\alpha>-1, \tau_{n}^{\alpha+1}=(\alpha+1)\left(s_{n}^{\alpha}-s_{n}^{\alpha+1}\right)$.

Lemma 3 ([6]). For $\alpha>-1, n \Delta \tau_{n}^{\alpha+1}=(\alpha+1)\left(\tau_{n}^{\alpha}-\tau_{n}^{\alpha+1}\right)$.

Lemma 4 ([13]). For $-1<\alpha<\beta,(A)(C, \alpha) \subset(A)(C, \beta)$. 
Lemma 5 ([7]). Let $\alpha>-1$. For any integer $m \geq 2$,

$$
(n \Delta)_{m} \tau_{n}^{\alpha+m}=\sum_{j=1}^{m}(-1)^{j+1} A_{m}^{(j)}(\alpha) n \Delta \tau_{n}^{(\alpha+j)},
$$

where

$$
A_{m}^{(j)}(\alpha)=a_{m}^{(j-1)}(\alpha)+a_{m}^{(j)}(\alpha), \quad a_{m}^{(0)}(\alpha)=0,
$$

and

$$
a_{m}^{(j)}(\alpha)=\prod_{k=j+1}^{m}(\alpha+k)\left[\sum_{\substack{j+1 \leq t_{1}, t_{2}, \ldots, t_{j-1} \leq m \\ r<s \Rightarrow t_{r} \leq t_{s}}}\left(\alpha+t_{1}\right)\left(\alpha+t_{2}\right) \ldots\left(\alpha+t_{j-1}\right)\right]
$$

where $j=1,2,3, \ldots, m$.

Lemma 6 ([7]). Let $\alpha>-1$. For any integer $m \geq 2$,

$$
(\alpha+j) A_{m}^{(j-1)}(\alpha+1)+(\alpha+j+1) A_{m}^{(j)}(\alpha+1)=A_{m+1}^{(j)}(\alpha),
$$

where $A_{m}^{(j)}(\alpha)$ is as in Lemma 5 .

Lemma 7 ([15]). For $\alpha>-1, \sigma_{n}\left(s^{\alpha}\right)=\frac{1}{\alpha+1} s_{n}^{\alpha+1}+\left(1-\frac{1}{\alpha+1}\right) \sigma_{n}\left(s^{\alpha+1}\right)$.

Lemma 8. i) For all $\lambda>1$ and large enough $n$, that is, when $[\lambda n]>n$,

$$
\begin{aligned}
s_{n}^{\alpha}-s_{n}^{\alpha+1}= & \frac{\alpha}{\alpha+1}\left[\frac{[\lambda n]+1}{[\lambda n]-n}\left(\sigma_{[\lambda n]}\left(s^{\alpha+1}\right)-\sigma_{n}\left(s^{\alpha+1}\right)\right)+\left(\sigma_{n}\left(s^{\alpha+1}\right)-s_{n}^{\alpha+1}\right)\right] \\
& +\frac{1}{\alpha} \frac{[\lambda n]+1}{[\lambda n]-n}\left(s_{[\lambda n]}^{\alpha+1}-s_{n}^{\alpha+1}\right)-\frac{1}{[\lambda n]-n} \sum_{k=n+1}^{[\lambda n]}\left(s_{k}^{\alpha}-s_{n}^{\alpha}\right)
\end{aligned}
$$

ii) For all $0<\lambda<1$ and large enough $n$, that is, when $n>[\lambda n]$,

$$
\begin{aligned}
s_{n}^{\alpha}-s_{n}^{\alpha+1}= & \frac{\alpha}{\alpha+1}\left[\frac{[\lambda n]+1}{n-[\lambda n]}\left(\sigma_{n}\left(s^{\alpha+1}\right)-\sigma_{[\lambda n]}\left(s^{\alpha+1}\right)\right)+\left(\sigma_{n}\left(s^{\alpha+1}\right)-s_{n}^{\alpha+1}\right)\right] \\
& +\frac{1}{\alpha} \frac{[\lambda n]+1}{n-[\lambda n]}\left(s_{n}^{\alpha+1}-s_{[\lambda n]}^{\alpha+1}\right)-\frac{1}{n-[\lambda n]} \sum_{k=[\lambda n]+1}^{n}\left(s_{n}^{\alpha}-s_{k}^{\alpha}\right),
\end{aligned}
$$

where $[\lambda n]$ denotes the integer part of the product $\lambda n$.

Proof. Let $\tau_{n,[\lambda n]}^{\alpha}=\frac{1}{[\lambda n]-n} \sum_{k=n+1}^{[\lambda n]} s_{k}^{\alpha}$. Then we have,

$$
\tau_{n,[\lambda n]}^{\alpha}-s_{n}^{\alpha+1}=\frac{1}{[\lambda n]-n} \sum_{k=n+1}^{[\lambda n]} s_{k}^{\alpha}-s_{n}^{\alpha+1}
$$




$$
\begin{aligned}
& =\frac{1}{[\lambda n]-n}\left(\sum_{k=0}^{[\lambda n]} s_{k}^{\alpha}-\sum_{k=0}^{n} s_{k}^{\alpha}\right)-s_{n}^{\alpha+1} \\
& =\frac{[\lambda n]+1}{([\lambda n]-n)([\lambda n]+1)} \sum_{k=0}^{n} s_{k}^{\alpha}-\frac{n+1}{([\lambda n]-n)(n+1)} \sum_{k=0}^{n} s_{k}^{\alpha}-s_{n}^{\alpha+1} \\
& =\frac{1}{[\lambda n]-n}\left(([\lambda n]+1) \sigma_{[\lambda n]}\left(s^{\alpha}\right)-(n+1) \sigma_{n}\left(s^{\alpha}\right)\right)-s_{n}^{\alpha+1}
\end{aligned}
$$

By lemma 7, we have

$$
\begin{aligned}
\tau_{n,[\lambda n]}^{\alpha}-s_{n}^{\alpha+1}= & \frac{1}{[\lambda n]-n}\left(([\lambda n]+1)\left(\frac{\alpha}{\alpha+1} \sigma_{[\lambda n]}\left(s^{\alpha+1}\right)+\frac{1}{\alpha+1} s_{[\lambda n]}^{\alpha+1}\right)\right. \\
& \left.-(n+1)\left(\frac{\alpha}{\alpha+1} \sigma_{n}\left(s^{\alpha+1}\right)+\frac{1}{\alpha+1} s_{n}^{\alpha+1}\right)\right)-s_{n}^{\alpha+1} \\
= & \frac{1}{[\lambda n]-n}\left(\frac{\alpha([\lambda n]+1)}{\alpha+1} \sigma_{[\lambda n]}\left(s^{\alpha+1}\right)+\frac{[\lambda n]+1}{\alpha+1} s_{[\lambda n]}^{\alpha+1}\right. \\
& \left.-\frac{(n+1) \alpha}{\alpha+1} \sigma_{n}\left(s^{\alpha+1}\right)-\frac{n+1}{\alpha+1} s_{n}^{\alpha+1}-([\lambda n]-n) s_{n}^{\alpha+1}\right) .
\end{aligned}
$$

or

$$
\begin{aligned}
s_{n}^{\alpha}-s_{n}^{\alpha+1}= & s_{n}^{\alpha}-\tau_{n,[\lambda n]}^{\alpha}+\tau_{n,[\lambda n]}^{\alpha}-s_{n}^{\alpha+1} \\
= & s_{n}^{\alpha}-\tau_{n,[\lambda n]}^{\alpha}+\frac{1}{[\lambda n]-n}\left(\frac{\alpha([\lambda n]+1)}{\alpha+1} \sigma_{[\lambda n]}\left(s^{\alpha+1}\right)+\frac{[\lambda n]+1}{\alpha+1} s_{[\lambda n]}^{\alpha+1}\right. \\
& \left.-\frac{(n+1) \alpha}{\alpha+1} \sigma_{n}\left(s^{\alpha+1}\right)-\frac{\alpha[\lambda n]-\alpha n+[\lambda n]+1}{\alpha+1} s_{n}^{\alpha+1}\right) \\
= & s_{n}^{\alpha}-\frac{1}{[\lambda n]-n} \sum_{k=n+1}^{[\lambda n]} s_{k}^{\alpha}+\frac{1}{[\lambda n]-n}\left(\frac{\alpha([\lambda n]+1)}{\alpha+1} \sigma_{[\lambda n]}\left(s^{\alpha+1}\right)\right. \\
& \left.+\frac{[\lambda n]+1}{\alpha+1} s_{[\lambda n]}^{\alpha+1}-\frac{\alpha(n+1)}{\alpha+1} \sigma_{n}\left(s^{\alpha+1}\right)-\frac{\alpha[\lambda n]-\alpha n+[\lambda n]+1}{\alpha+1} s_{n}^{\alpha+1}\right)
\end{aligned}
$$

We finally have

$$
\begin{aligned}
s_{n}^{\alpha}-s_{n}^{\alpha+1}= & \frac{1}{[\lambda n]-n}\left(\frac{\alpha([\lambda n]+1)}{\alpha+1} \sigma_{[\lambda n]}\left(s^{\alpha+1}\right)+\frac{[\lambda n]+1}{\alpha+1} s_{[\lambda n]}^{\alpha+1}\right. \\
& \left.-\frac{\alpha(n+1)}{\alpha+1} \sigma_{n}\left(s^{\alpha+1}\right)-\frac{\alpha[\lambda n]-\alpha n+[\lambda n]+1}{\alpha+1} s_{n}^{\alpha+1}\right)
\end{aligned}
$$




$$
\begin{aligned}
& -\frac{1}{[\lambda n]-n}\left(\sum_{k=n+1}^{[\lambda n]} s_{k}^{\alpha}-([\lambda n]-n) s_{n}^{\alpha}\right) \\
& =\frac{1}{[\lambda n]-n}\left(\frac{\alpha([\lambda n]+1)}{\alpha+1} \sigma_{[\lambda n]}\left(s^{\alpha+1}\right)-\frac{\alpha(n+1)}{\alpha+1} \sigma_{n}\left(s^{\alpha+1}\right)\right. \\
& +\frac{\alpha([\lambda n]+1)}{\alpha+1} \sigma_{n}\left(s^{\alpha+1}\right)-\frac{\alpha([\lambda n]+1)}{\alpha+1} \sigma_{n}\left(s^{\alpha+1}\right) \\
& \left.+\frac{[\lambda n]+1}{\alpha+1} s_{[\lambda n]}^{\alpha+1}-\frac{\alpha[\lambda n]-\alpha n+[\lambda n]+1}{\alpha+1} s_{n}^{\alpha+1}\right) \\
& -\frac{1}{[\lambda n]-n}\left(\sum_{k=n+1}^{[\lambda n]} s_{k}^{\alpha}-\sum_{k=n+1}^{[\lambda n]} s_{n}^{\alpha}\right) \\
& =\frac{1}{[\lambda n]-n}\left[\frac{\alpha([\lambda n]+1)}{\alpha+1}\left(\sigma_{[\lambda n]}\left(s^{\alpha+1}\right)-\sigma_{n}\left(s^{\alpha+1}\right)\right)\right. \\
& -\frac{\alpha}{\alpha+1}\left((n+1) \sigma_{n}\left(s^{\alpha+1}\right)-([\lambda n]+1) \sigma_{n}\left(s^{\alpha+1}\right)\right) \\
& \left.+\frac{[\lambda n]+1}{\alpha+1} s_{[\lambda n]}^{\alpha+1}-\frac{\alpha[\lambda n]-\alpha n+[\lambda n]+1}{\alpha+1} s_{n}^{\alpha+1}\right] \\
& -\frac{1}{[\lambda n]-n} \sum_{k=n+1}^{[\lambda n]}\left(s_{k}^{\alpha}-s_{n}^{\alpha}\right) \\
& =\frac{1}{[\lambda n]-n}\left[\frac{\alpha([\lambda n]+1)}{\alpha+1}\left(\sigma_{[\lambda n]}\left(s^{\alpha+1}\right)-\sigma_{n}\left(s^{\alpha+1}\right)\right)\right. \\
& -\frac{\alpha(n-[\lambda n])}{\alpha+1} \sigma_{n}\left(s^{\alpha+1}\right)+\frac{[\lambda n]+1}{\alpha+1} s_{[\lambda n]}^{\alpha+1}+\frac{\alpha(n-[\lambda n])}{\alpha+1} s_{n}^{\alpha+1} \\
& \left.-\frac{[\lambda n]+1}{\alpha+1} s_{n}^{\alpha+1}\right]-\frac{1}{[\lambda n]-n} \sum_{k=n+1}^{[\lambda n]}\left(s_{k}^{\alpha}-s_{n}^{\alpha}\right) \\
& =\frac{\alpha}{\alpha+1}\left[\frac{[\lambda n]+1}{[\lambda n]-n}\left(\sigma_{[\lambda n]}\left(s^{\alpha+1}\right)-\sigma_{n}\left(s^{\alpha+1}\right)\right)\right. \\
& \left.+\left(\sigma_{n}\left(s^{\alpha+1}\right)-s_{n}^{\alpha+1}\right)\right]+\frac{1}{\alpha} \frac{[\lambda n]+1}{[\lambda n]-n}\left(s_{[\lambda n]}^{\alpha+1}-s_{n}^{\alpha+1}\right) \\
& -\frac{1}{[\lambda n]-n} \sum_{k=n+1}^{[\lambda n]}\left(s_{k}^{\alpha}-s_{n}^{\alpha}\right) \text {. }
\end{aligned}
$$

This completes the proof.

The proof for ii) is similar to that of i). 


\section{Proof of TheOREM 7}

By hypothesis, $s_{n}^{\alpha+1} \rightarrow s(A)$. By Lemma 4, we have $s_{n}^{\alpha+1} \rightarrow s(A), s_{n}^{\alpha+2} \rightarrow$ $s(A), \ldots, s_{n}^{\alpha+m} \rightarrow s(A)$ where $m$ is any positive integer. Hence, by Lemma 2, we get

$$
\begin{aligned}
(\alpha+2)\left(s_{n}^{\alpha+1}-s_{n}^{\alpha+2}\right) & =\tau_{n}^{\alpha+2} \\
(\alpha+3)\left(s_{n}^{\alpha+2}-s_{n}^{\alpha+3}\right) & =\tau_{n}^{\alpha+3} \\
\vdots & \\
(\alpha+m+1)\left(s_{n}^{\alpha+m}-s_{n}^{\alpha+m+1}\right) & =\quad \tau_{n}^{\alpha+m+1} .
\end{aligned}
$$

Since $s_{n}^{\alpha+k} \rightarrow s(A)$ for $k=1,2, \ldots, m+1$, we have

$$
\begin{aligned}
\tau_{n}^{\alpha+2} & \rightarrow 0(A) \\
\tau_{n}^{\alpha+3} & \rightarrow 0(A) \\
\vdots & \\
\tau_{n}^{\alpha+m+1} & \rightarrow 0(A)
\end{aligned}
$$

and by Lemma 3 ,

$$
\begin{aligned}
&(\alpha+3)\left(\tau_{n}^{\alpha+2}-\tau_{n}^{\alpha+3}\right)=n \Delta \tau_{n}^{\alpha+3} \rightarrow 0(A) \\
&(\alpha+4)\left(\tau_{n}^{\alpha+3}-\tau_{n}^{\alpha+4}\right)=n \Delta \tau_{n}^{\alpha+4} \rightarrow 0(A) \\
& \vdots \\
&(\alpha+m+1)\left(\tau_{n}^{\alpha+m}-\tau_{n}^{\alpha+m+1}\right)=n \Delta \tau_{n}^{\alpha+m+1} \rightarrow 0(A) .
\end{aligned}
$$

Since

$$
(n \Delta)_{m} \tau_{n}^{\alpha+m} \geq-H
$$

then

$$
(n \Delta)_{m} \tau_{n}^{\alpha+m+j} \geq-H_{1}
$$

for $j=1, \ldots, m-1$, by Lemma 5 , we have

$$
\begin{aligned}
(n \Delta)_{m-1} \tau_{n}^{\alpha+m+1}= & \sum_{j=1}^{m-1}(-1)^{j+1} A_{m-1}^{j}(\alpha+2) n \Delta \tau_{n}^{\alpha+2+j} \\
= & A_{m-1}^{1}(\alpha+2) n \Delta \tau_{n}^{\alpha+3}-A_{m-1}^{2}(\alpha+2) n \Delta \tau_{n}^{\alpha+4}+\ldots \\
& +(-1)^{m} A_{m-1}^{m-1}(\alpha+2) n \Delta \tau_{n}^{\alpha+m+1} .
\end{aligned}
$$

For $j=1, \ldots, m-1$ we have $n \Delta \tau_{n}^{\alpha+2+j} \rightarrow 0(A)$. Hence, we get

$$
(n \Delta)_{m-1} \tau_{n}^{\alpha+m+1} \rightarrow 0(A)
$$


It follows from (5.3) that

$$
(n \Delta)_{m-1} \tau_{n}^{\alpha+m+1}=o(1)
$$

by Theorem 3. By Lemma 3, we obtain

$$
(\alpha+m+1)\left((n \Delta)_{m-1} \tau_{n}^{\alpha+m}-(n \Delta)_{m-1} \tau_{n}^{\alpha+m+1}\right)=(n \Delta)_{m} \tau_{n}^{\alpha+m+1} .
$$

Substituting (5.3) and (5.5) into (5.6), we have

$$
(n \Delta)_{m-1} \tau_{n}^{\alpha+m} \geq-H_{2} .
$$

Since

$$
\begin{aligned}
(n \Delta)_{m-2} \tau_{n}^{\alpha+m}= & \sum_{j=1}^{m-2}(-1)^{j+1} A_{m-2}^{j}(\alpha+2) n \Delta \tau_{n}^{\alpha+2+j} \\
= & A_{m-2}^{1}(\alpha+2) n \Delta \tau_{n}^{\alpha+3}-A_{m-2}^{2}(\alpha+2) n \Delta \tau_{n}^{\alpha+4}+\ldots \\
& +(-1)^{m-2} A_{m-2}^{m-2}(\alpha+2) n \Delta \tau_{n}^{\alpha+m}
\end{aligned}
$$

by Lemma 5, we have

$$
(n \Delta)_{m-2} \tau_{n}^{\alpha+m} \rightarrow 0(A) .
$$

From (5.7) and (5.8), we obtain, by Theorem 3,

$$
(n \Delta)_{m-2} \tau_{n}^{\alpha+m}=o(1) .
$$

By Lemma 3, we obtain

$$
(\alpha+m)\left((n \Delta)_{m-2} \tau_{n}^{\alpha+m-1}-(n \Delta)_{m-2} \tau_{n}^{\alpha+m}\right)=(n \Delta)_{m-1} \tau_{n}^{\alpha+m} .
$$

Substituting (5.7) and (5.9) into (5.10), we have

$$
(n \Delta)_{m-2} \tau_{n}^{\alpha+m-1} \geq-H_{3} .
$$

Since

$$
\begin{aligned}
(n \Delta)_{m-3} \tau_{n}^{\alpha+m-1}= & \sum_{j=1}^{m-3}(-1)^{j+1} A_{m-3}^{j}(\alpha+2) n \Delta \tau_{n}^{\alpha+2+j} \\
= & A_{m-3}^{1}(\alpha+2) n \Delta \tau_{n}^{\alpha+3}-A_{m-3}^{2}(\alpha+2) n \Delta \tau_{n}^{\alpha+4}+\ldots \\
& +(-1)^{m-2} A_{m-3}^{m-3}(\alpha+2) n \Delta \tau_{n}^{\alpha+m-2}
\end{aligned}
$$

by Lemma 3, we have

$$
(n \Delta)_{m-3} \tau_{n}^{\alpha+m-1} \rightarrow 0(A)
$$

From (5.11) and (5.12), we have, by Theorem 3,

$$
(n \Delta)_{m-3} \tau_{n}^{\alpha+m-1}=o(1) .
$$

By Lemma 3, we obtain

$$
(\alpha+m-1)\left((n \Delta)_{m-3} \tau_{n}^{\alpha+m-2}-(n \Delta)_{m-3} \tau_{n}^{\alpha+m-1}\right)=(n \Delta)_{m-2} \tau_{n}^{\alpha+m-1} .
$$


Substituting (5.11) and (5.13) into (5.14), we have

$$
(n \Delta)_{m-3} \tau_{n}^{\alpha+m-2} \geq-H_{4} .
$$

Continuing in this way, we obtain

$$
(n \Delta)_{2} \tau_{n}^{\alpha+4} \rightarrow 0(A)
$$

and

$$
(n \Delta)_{3} \tau_{n}^{\alpha+4} \geq-H_{5} .
$$

From (5.16) and (5.17), we have, by Theorem 3,

$$
(n \Delta)_{2} \tau_{n}^{\alpha+4}=o(1) .
$$

By Lemma 3, we obtain

$$
(\alpha+4)\left((n \Delta)_{2} \tau_{n}^{\alpha+3}-(n \Delta)_{2} \tau_{n}^{\alpha+4}\right)=(n \Delta)_{3} \tau_{n}^{\alpha+4} .
$$

Substituting (5.17) and (5.18) into (5.19), we have

$$
(n \Delta)_{2} \tau_{n}^{\alpha+3} \geq-H_{6} .
$$

From $n \Delta \tau_{n}^{\alpha+3} \rightarrow 0(A)$ and (5.20), we have, by Theorem 3,

$$
n \Delta \tau_{n}^{\alpha+3}=o(1) .
$$

By Lemma 3, we obtain

$$
(\alpha+3)\left(n \Delta \tau_{n}^{\alpha+2}-n \Delta \tau_{n}^{\alpha+3}\right)=(n \Delta)_{2} \tau_{n}^{\alpha+3} .
$$

Substituting (5.20) and (5.21) into (5.22), we have

$$
n \Delta \tau_{n}^{\alpha+2} \geq-H_{7} .
$$

It follows from (5.1) and (5.23) by Theorem 1, we get

$$
\tau_{n}^{\alpha+2}=o(1)
$$

Substituting (5.23) and (5.24) into

$$
(\alpha+2)\left(\tau_{n}^{\alpha+1}-\tau_{n}^{\alpha+2}\right)=n \Delta \tau_{n}^{\alpha+2}
$$

we have

Since $s_{n}^{\alpha+1} \rightarrow s(A)$ and

$$
\tau_{n}^{\alpha+1} \geq-H_{8}
$$

$$
\tau_{n}^{\alpha+1}=n \Delta s_{n}^{\alpha+1} \geq-H_{8},
$$

we obtain, by Theorem 3,

$$
s_{n}^{\alpha+1} \rightarrow s
$$

Now we need to show that

$$
\tau_{n}^{\alpha}=n \Delta s_{n}^{\alpha} \geq-C
$$

for some constant $C$. 
From (5.2), by Lemma 5, we have, for $j=1, \ldots, m-1$,

$$
\begin{aligned}
(n \Delta)_{m-1} \tau_{n}^{\alpha+m}= & \sum_{j=1}^{m-1}(-1)^{j+1} A_{m-1}^{j}(\alpha+1) n \Delta \tau_{n}^{\alpha+1+j} \\
= & A_{m-1}^{1}(\alpha+1) n \Delta \tau_{n}^{\alpha+2}-A_{m-1}^{2}(\alpha+1) n \Delta \tau_{n}^{\alpha+3}+\ldots \\
& +(-1)^{m} A_{m-1}^{m-1}(\alpha+1) n \Delta \tau_{n}^{\alpha+m}
\end{aligned}
$$

We have $n \Delta \tau_{n}^{\alpha+1+j} \rightarrow 0(A)$ for $j=1, \ldots, m-1$. Hence, we get

$$
(n \Delta)_{m-1} \tau_{n}^{\alpha+m} \rightarrow 0(A) \text {. }
$$

It follows from (5.2) that

$$
(n \Delta)_{m-1} \tau_{n}^{\alpha+m}=o(1)
$$

by Theorem 3. By Lemma 3, we obtain

$$
(\alpha+m)\left((n \Delta)_{m-1} \tau_{n}^{\alpha+m-1}-(n \Delta)_{m-1} \tau_{n}^{\alpha+m}\right)=(n \Delta)_{m} \tau_{n}^{\alpha+m} .
$$

Substituting (5.2) and (5.29) into (5.30), we have

$$
(n \Delta)_{m-1} \tau_{n}^{\alpha+m-1} \geq-H_{10}
$$

Since

$$
\begin{aligned}
(n \Delta)_{m-2} \tau_{n}^{\alpha+m-1}= & \sum_{j=1}^{m-2}(-1)^{j+1} A_{m-2}^{j}(\alpha+1) n \Delta \tau_{n}^{\alpha+1+j} \\
= & A_{m-2}^{1}(\alpha+1) n \Delta \tau_{n}^{\alpha+2}-A_{m-2}^{2}(\alpha+1) n \Delta \tau_{n}^{\alpha+3}+\ldots \\
& +(-1)^{m-2} A_{m-2}^{m-2}(\alpha+1) n \Delta \tau_{n}^{\alpha+m-1}
\end{aligned}
$$

by Lemma 5, we have

$$
(n \Delta)_{m-2} \tau_{n}^{\alpha+m-1} \rightarrow 0(A) .
$$

From (5.31) and (5.32), we obtain, by Theorem 3,

$$
(n \Delta)_{m-2} \tau_{n}^{\alpha+m-1}=o(1) .
$$

By Lemma 3, we obtain

$$
(\alpha+m-1)\left((n \Delta)_{m-2} \tau_{n}^{\alpha+m-2}-(n \Delta)_{m-2} \tau_{n}^{\alpha+m-1}\right)=(n \Delta)_{m-1} \tau_{n}^{\alpha+m-1} .
$$

Substituting (5.31) and (5.33) into (5.34), we have

$$
(n \Delta)_{m-2} \tau_{n}^{\alpha+m-2} \geq-H_{11} \text {. }
$$

Since

$$
\begin{aligned}
(n \Delta)_{m-3} \tau_{n}^{\alpha+m-2} & =\sum_{j=1}^{m-3}(-1)^{j+1} A_{m-3}^{j}(\alpha+1) n \Delta \tau_{n}^{\alpha+1+j} \\
& =A_{m-3}^{1}(\alpha+1) n \Delta \tau_{n}^{\alpha+2}-A_{m-3}^{2}(\alpha+1) n \Delta \tau_{n}^{\alpha+3}+\ldots
\end{aligned}
$$




$$
+(-1)^{m-2} A_{m-3}^{m-3}(\alpha+1) n \Delta \tau_{n}^{\alpha+m-3}
$$

by Lemma 3, we have

$$
(n \Delta)_{m-3} \tau_{n}^{\alpha+m-2} \rightarrow 0(A)
$$

From (5.35) and (5.36), we have, by Theorem 3,

$$
(n \Delta)_{m-3} \tau_{n}^{\alpha+m-2}=o(1) .
$$

By Lemma 3, we obtain

$$
(\alpha+m-2)\left((n \Delta)_{m-3} \tau_{n}^{\alpha+m-3}-(n \Delta)_{m-3} \tau_{n}^{\alpha+m-2}\right)=(n \Delta)_{m-2} \tau_{n}^{\alpha+m-2} .
$$

Substituting (5.35) and (5.37) into (5.38), we have

$$
(n \Delta)_{m-3} \tau_{n}^{\alpha+m-3} \geq-H_{12}
$$

Continuing in this way, we obtain

$$
(n \Delta)_{2} \tau_{n}^{\alpha+3} \rightarrow 0(A)
$$

and

$$
(n \Delta)_{3} \tau_{n}^{\alpha+3} \geq-H_{13}
$$

From (5.40) and (5.41), we have, by Theorem 3,

$$
(n \Delta)_{2} \tau_{n}^{\alpha+3}=o(1) \text {. }
$$

By Lemma 3, we obtain

$$
(\alpha+3)\left((n \Delta)_{2} \tau_{n}^{\alpha+2}-(n \Delta)_{2} \tau_{n}^{\alpha+3}\right)=(n \Delta)_{3} \tau_{n}^{\alpha+3} .
$$

Substituting (5.41) and (5.42) into (5.43), we have

$$
(n \Delta)_{2} \tau_{n}^{\alpha+2} \geq-H_{14}
$$

From $n \Delta \tau_{n}^{\alpha+2} \rightarrow 0(A)$ and (5.44), we have, by Theorem 3,

$$
n \Delta \tau_{n}^{\alpha+2}=o(1) \text {. }
$$

By Lemma 3, we obtain

$$
(\alpha+2)\left(n \Delta \tau_{n}^{\alpha+1}-n \Delta \tau_{n}^{\alpha+2}\right)=(n \Delta)_{2} \tau_{n}^{\alpha+2} .
$$

Substituting (5.44) and (5.45) into (5.46), we have

$$
n \Delta \tau_{n}^{\alpha+1} \geq-H_{15}
$$

Substituting (5.47), (5.25) and (5.27) into

$$
(\alpha+1)\left(\tau_{n}^{\alpha}-\tau_{n}^{\alpha+1}\right)=n \Delta \tau_{n}^{\alpha+1}
$$

we have

$$
\tau_{n}^{\alpha}=n \Delta s_{n}^{\alpha} \geq-H_{16} .
$$

By Lemma 8 i) and (5.48), we have

$$
s_{n}^{\alpha}-s_{n}^{\alpha+1}=\frac{\alpha}{\alpha+1}\left[\frac{[\lambda n]+1}{[\lambda n]-n}\left(\sigma_{[\lambda n]}\left(s^{\alpha+1}\right)-\sigma_{n}\left(s^{\alpha+1}\right)\right)+\left(\sigma_{n}\left(s^{\alpha+1}\right)-s_{n}^{\alpha+1}\right)\right]
$$




$$
\begin{aligned}
& +\frac{1}{\alpha} \frac{[\lambda n]+1}{[\lambda n]-n}\left(s_{\lambda n}^{\alpha+1}-s_{n}^{\alpha+1}\right)-\frac{1}{[\lambda n]-n} \sum_{k=n+1}^{[\lambda n]} \sum_{j=n+1}^{k} \Delta s_{j}^{\alpha} \\
\leq & \frac{\alpha}{\alpha+1}\left[\frac{[\lambda n]+1}{[\lambda n]-n}\left(\sigma_{[\lambda n]}\left(s^{\alpha+1}\right)-\sigma_{n}\left(s^{\alpha+1}\right)\right)+\left(\sigma_{n}\left(s^{\alpha+1}\right)-s_{n}^{\alpha+1}\right)\right] \\
& +\frac{1}{\alpha} \frac{[\lambda n]+1}{[\lambda n]-n}\left(s_{\lambda n}^{\alpha+1}-s_{n}^{\alpha+1}\right)+\frac{1}{[\lambda n]-n} \sum_{k=n+1}^{[\lambda n]} \sum_{j=n+1}^{k} \frac{H}{j} \\
\leq & \frac{\alpha}{\alpha+1}\left[\frac{[\lambda n]+1}{[\lambda n]-n}\left(\sigma_{[\lambda n]}\left(s^{\alpha+1}\right)-\sigma_{n}\left(s^{\alpha+1}\right)\right)+\left(\sigma_{n}\left(s^{\alpha+1}\right)-s_{n}^{\alpha+1}\right)\right] \\
& +\frac{1}{\alpha} \frac{[\lambda n]+1}{[\lambda n]-n}\left(s_{\lambda n}^{\alpha+1}-s_{n}^{\alpha+1}\right)+H \log \frac{[\lambda n]}{n}
\end{aligned}
$$

Taking the limsup of both sides, we get

$$
\begin{aligned}
\limsup _{n \rightarrow \infty}\left(s_{n}^{\alpha}-s_{n}^{\alpha+1}\right) & \leq \limsup _{n \rightarrow \infty}\left\{\frac { \alpha } { \alpha + 1 } \left[\frac{[\lambda n]+1}{[\lambda n]-n}\left(\sigma_{[\lambda n]}\left(s^{\alpha+1}\right)-\sigma_{n}\left(s^{\alpha+1}\right)\right)\right.\right. \\
& \left.\left.+\left(\sigma_{n}\left(s^{\alpha+1}\right)-s_{n}^{\alpha+1}\right)\right]+\frac{1}{\alpha} \frac{[\lambda n]+1}{[\lambda n]-n}\left(s_{\lambda n}^{\alpha+1}-s_{n}^{\alpha+1}\right)\right\}+H \log \lambda,
\end{aligned}
$$

where $H>0$. Since

$$
\frac{[\lambda n]+1}{[\lambda n]-n} \leq \frac{2 \lambda}{\lambda-1}
$$

for $\lambda>1$ and sufficiently large $n$ and $s_{n}^{\alpha+1} \rightarrow s$, we have

$$
\lim _{\lambda \rightarrow 1^{+}} \limsup _{n \rightarrow \infty}\left(s_{n}^{\alpha}-s_{n}^{\alpha+1}\right) \leq 0
$$

By Lemma 8 ii) and (5.48), we have

$$
\begin{aligned}
s_{n}^{\alpha}-s_{n}^{\alpha+1}= & \frac{\alpha}{\alpha+1}\left[\frac{[\lambda n]+1}{n-[\lambda n]}\left(\sigma_{n}\left(s^{\alpha+1}\right)-\sigma_{[\lambda n]}\left(s^{\alpha+1}\right)\right)+\left(\sigma_{n}\left(s^{\alpha+1}\right)-s_{n}^{\alpha+1}\right)\right] \\
& +\frac{1}{\alpha} \frac{[\lambda n]+1}{n-[\lambda n]}\left(s_{n}^{\alpha+1}-s_{[\lambda n]}^{\alpha+1}\right)+\frac{1}{n-[\lambda n]} \sum_{k=[\lambda n]+1}^{n} \sum_{j=n+1}^{k} \Delta s_{j}^{\alpha} \\
\geq & \frac{\alpha}{\alpha+1}\left[\frac{[\lambda n]+1}{n-[\lambda n]}\left(\sigma_{n}\left(s^{\alpha+1}\right)-\sigma_{[\lambda n]}\left(s^{\alpha+1}\right)\right)+\left(\sigma_{n}\left(s^{\alpha+1}\right)-s_{n}^{\alpha+1}\right)\right] \\
& +\frac{1}{\alpha} \frac{[\lambda n]+1}{n-[\lambda n]}\left(s_{n}^{\alpha+1}-s_{\lambda n}^{\alpha+1}\right)+\frac{1}{n-[\lambda n]} \sum_{k=[\lambda n]+1}^{n} \sum_{j=n+1}^{k} \frac{H}{j} \\
\geq & \frac{\alpha}{\alpha+1}\left[\frac{[\lambda n]+1}{n-[\lambda n]}\left(\sigma_{n}\left(s^{\alpha+1}\right)-\sigma_{[\lambda n]}\left(s^{\alpha+1}\right)\right)+\left(\sigma_{n}\left(s^{\alpha+1}\right)-s_{n}^{\alpha+1}\right)\right]
\end{aligned}
$$




$$
+\frac{1}{\alpha} \frac{[\lambda n]+1}{n-[\lambda n]}\left(s_{n}^{\alpha+1}-s_{[\lambda n]}^{\alpha+1}\right)-H \log \frac{[\lambda n]}{n}
$$

Taking the liminf of both sides, we get

$$
\begin{aligned}
\liminf _{n \rightarrow \infty}\left(s_{n}^{\alpha}-s_{n}^{\alpha+1}\right) & \geq \liminf _{n \rightarrow \infty}\left\{\frac { \alpha } { \alpha + 1 } \left[\frac{[\lambda n]+1}{n-[\lambda n]}\left(\sigma_{n}\left(s^{\alpha+1}\right)-\sigma_{[\lambda n]}\left(s^{\alpha+1}\right)\right)\right.\right. \\
& \left.\left.+\left(\sigma_{n}\left(s^{\alpha+1}\right)-s_{n}^{\alpha+1}\right)\right]+\frac{1}{\alpha} \frac{[\lambda n]+1}{n-[\lambda n]}\left(s_{n}^{\alpha+1}-s_{[\lambda n]}^{\alpha+1}\right)\right\}+H \log \lambda
\end{aligned}
$$

where $H>0$. Since

$$
\frac{[\lambda n]+1}{n-[\lambda n]} \leq \frac{2 \lambda}{1-\lambda}
$$

for $0<\lambda<1$ and sufficiently large $n$ and $s_{n}^{\alpha+1} \rightarrow s$, we have

$$
\lim _{\lambda \rightarrow 1^{+}} \liminf _{n \rightarrow \infty}\left(s_{n}^{\alpha}-s_{n}^{\alpha+1}\right) \geq 0 .
$$

Combining (5.50) and (5.52) provides

$$
\lim s_{n}^{\alpha}=\lim s_{n}^{\alpha+1} .
$$

This completes the proof.

We like to note that we used $H$ to denote a constant, possibly different at each occurrence above.

\section{REFERENCES}

[1] Abel, N. H., "Recherches sur la série 1+ $\frac{m}{1} x+\frac{m(m-1)}{1.2} x^{2}+\ldots$," J. für Math., vol. 1, pp. 311-319, 1826.

[2] A. Amir, "On a converse of Abel's theorem," Proc. Am. Math. Soc., vol. 3, pp. 244-256, 1952.

[3] J. Boos, Classical and modern methods in summability. New York, NY: Oxford University Press, 2000.

[4] D. Borwein, "Theorems on some methods of summability," Quart. J. Math., vol. 9, pp. 310-316, 1958.

[5] I. Çanak and Y. Erdem, "On Tauberian theorems for $(A)(C, \alpha)$ summability method," Appl. Math. Comput., vol. 218, no. 6, pp. 2829-2836, 2011.

[6] I. Çanak, Y. Erdem, and U. Totur, "Some Tauberian theorems for $(\mathrm{A})(C, \alpha)$ summability method," Math. Comput. Modelling, vol. 52, no. 5-6, pp. 738-743, 2010.

[7] Y. Erdem and I. Çanak, "A Tauberian theorem for $(A)(C, \alpha)$ summability," Comput. Math. Appl., vol. 60, no. 11, pp. 2920-2925, 2010.

[8] G. H. Hardy, "Theorems relating to the summability and convergence of slowly oscillating series," London. M. S. Proc., vol. 8, no. 2, pp. 301-320, 1910.

[9] G. H. Hardy and J. E. Littlewood, "Tauberian theorems concerning power series and Dirichlet's series whose coefficients are positive," London M. S. Proc., vol. 13, no. 2, pp. 174-191, 1914.

[10] E. Kogbetliantz, "Sur les séries absolument sommables par la méthode des moyennes arithmétiques," Bulletin sc. Math., vol. 49, no. 2, pp. 234-251, 1925.

[11] E. Kogbetliantz, "Sommation des séries et intégrales divergentes par les moyennes arithmétiques et typiques," Memorial Sci. Math., vol. 51, pp. 1-84, 1931.

[12] J. E. Littlewood, "The converse of Abel's theorem on power series," Lond. M. S. Proc., vol. 9, no. 2, pp. 434-448, 1911. 
[13] R. D. Lord, "On some relations between the Abel, Borel, and Cesàro methods of summation," Proceedings L. M. S., vol. 38, no. 2, pp. 241-256, 1934.

[14] Pati, T., "On Tauberian Theorems," in Sequences, Summability and Fourier Analysis, D. Rath and S. Nanda, Eds. Moscow: Narosa Publishing House, 2005, pp. 84-96.

[15] C. Rajagopal, "On a theorem connecting Borel and Cesaro summabilities," J. Indian Math. Soc., New Ser., vol. 24, pp. 433-442, 1961.

[16] O. Szász, "Generalization of two theorems of Hardy and Littlewood on power series," Duke Math. J., vol. 1, pp. 105-111, 1935.

[17] A. Tauber, "Ein Satz aus der Theorie der unendlichen Reihen," Monatsh. f. Math., vol. 8, pp. 273-277, 1897.

Authors' addresses

\section{Yilmaz Erdem}

Adnan Menderes University, Department of Economics and Finance, Aydin 09970 Turkey, Tel: +90 25677270 97, Fax: +902567727098

E-mail address: yerdemeadu.edu.tr; yilmazerdem2019@gmail.com

İbrahim Çanak

Ege University, Department of Mathematics, 35100 Izmir, Turkey

E-mail address: ibrahimcanak@yahoo.com 